Article

\title{
Low-Carbon Economical Dispacth of Electricity-Heat Integrated Energy System Considering Concentrating Solar Power and Heat Loss in Heat Pipe Network
}

\author{
Nenghan Zhang ${ }^{1 * \mathbb{D}}$, Yufeng Wang ${ }^{2}$, Xiyan Jian ${ }^{3}$ and Yibo Ding ${ }^{1}$ \\ 1 School of Electrical Engineering, Xi'an Jiaotong University, Xianning West Rd.28, Xi'an 710049, China; \\ dennygz@126.com (N.Z.); dyb0712@stu.xjtu.edu.cn (Y.D.) \\ 2 Jinhe Center for Economic Research, Xi'an Jiaotong University, Xianning West Rd.28, Xi'an 710049, China; \\ yfwangxjtu@163.com \\ 3 School of Electronic and Information Engineering, Xi'an Jiaotong University, Xianning West Rd.28, Xi'an \\ 710049, China; jxy803@stu.xjtu.edu.cn \\ * Correspondence: dennygz@126.com; Tel.: +86-156-1931-1297
}

\begin{abstract}
With the development of energy internet, integrated energy system can effectively reduce carbon emissions and improve the utilization of renewable energy. In this paper, a low-carbon optimal scheduling model of integrated energy system considering heat loss of heat network pipeline is proposed. Based on the study of concentrating solar power (CSP) plant and heat storage tank (HS), an optimal scheduling model is established, which takes system operation cost, environmental pollution and penalty cost of abandoning wind and solar energy as objectives. Through the analysis of example results, it is proved that the model proposed in this paper can achieve the goal of reliable, low-carbon and economic operation of the system. At the same time, it shows that CSP unit can reduce the operation cost of system and increase energy coupling and utilization.
\end{abstract}

Keywords: heat loss;integrated energy system;concentrating solar power;low-carbon;heat storage tank;economical dispatch

\section{Introduction}

With the energy and the environmental pollution intensify day by day, increasing the utilization rate of renewable energy and reducing the carbon emissions are highlighted. In integrated energy system (IES), energy conversion equipment such as CSP plant enriches the ways of utilizing renewable energy for IES and heat storage tank can store heat when there is waste heat in the system and release heat when the heat load is in short supply, thus reducing the waste heat of the system and further improving the economy of the system.

The integrated energy system has attracted a large number of researchers' attention. At present, the main research directions are to improve the energy efficiency of IES and minimize the operation cost. Reference [1] proposed a new multi energy flow calculation method for electric-gas-heat integrated energy system (EGH-IES) based on Newton Raphson method. Reference [2] proposed an IES collaborative scheduling model with power to gas (P2G) element and analyzed the economic benefits of wind power consumption, but the network constraints of thermal system were not considered within the model. Reference [3] proposed an optimal coordination model of combined cooling, heating and electric power system (CCHP) with different price rate structures and adopted neighborhood re-dispatch particle swarm optimization (NR-PSO) algorithm. Realizing collaborative dispatch of multi-district IES is vital for energy-saving and emission-reduction, where the first problem is the modeling of district heating and cooling network (DHCN). A general energy transfer model of the 
district heating network was proposed in [4] to solve the flow and temperature of the media in the heating network. Several methods for modeling the heat supply network were proposed in [5] and [6]. However, their models were simple and the degree of simulation was not meticulous. A relatively complete heat supply network model was established in [7] but it cannot be solved quickly and concisely. In order to unify the model of power network and heat supply network, the traditional heating system model has to assume a mixed temperature of backwater and fix the temperature of return water in the whole return water pipeline at this temperature. The traditional heat supply network model is not consistent with the actual thermal system, and will cause large calculation error. An operation optimization model of electricity-heat integrated energy system with heat supply network constraints was proposed in [8]. Although the temperature difference of backwater nodes is considered, the calculation model needs to establish a complex correlation matrix, which is slow in calculation and large in amount of calculation. In recent years, solar thermal power generation, as a new industry of concentrating solar power, has also been widely concerned. Reference [9] divided power generation of CSP into three parts: concentrating heat collection, heat storage and power generation, and established a power grid dispatching model with thermal storage. Reference [10] further constructed a two-stage stochastic scheduling model composed of day-ahead market and actual operation. Reference [11] constructed a photovoltaic cogeneration microgrid with demand response. The modeling method for CSP adopted in [10] and [11] is similar to thermal power units, which ignored the characteristics of heat accumulation during CSP unit startup.

In view of these problems, firstly, this paper studies the hydraulic and thermal models of the heat supply network, and puts forward the calculation model of the thermal system power flow considering the heat loss of the return water pipe network; then establishes a CSP unit model considering the start-up heat constraint, and analyzes the low-carbon economic operation optimization problem of the electricity-heat integrated energy system based on former research. The case study shows that the low-carbon economic dispatch model of integrated energy system established in this paper is correct and the solution results are effective. At the same time, CSP unit has certain potential in reducing the production cost of the system and promoting the consumption of renewable energy.

The remainder of this paper is organized as follows. Section II describes a CSP unit model considering the start-up heat constraint, district heat network (DHN) model considering heat loss and other units in IES. In Section III establishes a low-carbon economic dispatch model of IES. In Section IV, case studies are carried out to show the performance of the proposed model. Conclusions are given in Section V.

\section{IES Model}

\subsection{Model of CSP}

2.1.1. Heat balance of solar thermal power generation system

The three parts of CSP unit have the following thermal power balance relations

$$
\begin{gathered}
Q_{C S P, t}^{s f}=Q_{C S P, t}^{D}+Q_{C S P, t}^{i n} \\
\frac{Q_{C S P, t}^{\text {out }}}{\eta_{\text {out }}}-Q_{C S P, t}^{\text {in }} \eta_{\text {in }}=\Delta Q_{C S P, t} \\
Q_{C S P, t}^{\text {out }}=Q_{C S P, t}^{S U}+Q_{C S P, t}^{\text {gen }}
\end{gathered}
$$

Where, $Q_{C S P, t}^{s f}$ is the solar heat power received by photothermal unit of CSP at period $t ; Q_{C S P, t}^{D}$ represents the waste heat of CSP; $Q_{C S P, t}^{\text {in }}$ refers to the heat into the heat storage tank by the collector; $Q_{C S P, t}^{\text {out }}$ is the heat from the heat storage tank into the steam turbine generator; $\eta_{i n}$ and $\eta_{\text {out }}$ refer to the heat 
input efficiency and heat output efficiency of the heat storage tank respectively; $\Delta Q_{C S P, t}$ represents the change of heat storage tank during $t$ period; $Q_{C S P, t}^{S U}$ is the heat from the collector into the turbine and $Q_{C S P, t}^{g e n}$ refers to the heat generated by steam turbine.

Equation (3) indicates that part of the heat into the steam turbine is used for start-up and part of it is used for power generation. If the steam turbine has been started, it is directly used for power generation.

Heat balance equation of CSP can be obtained by simplifying (1) and (3):

$$
Q_{C S P, t}^{s f}=Q_{C S P, t}^{D}+Q_{C S P, t}^{i n}-Q_{C S P, t}^{o u t}+Q_{C S P, t}^{S U}+Q_{C S P, t}^{g e n}
$$

\subsubsection{Constraints of heat storage tank in CSP}

The heat inflow and outflow of heat storage tank of CSP unit meet the following constraints:

$$
\begin{aligned}
& 0 \leq Q_{C S P, t}^{\text {out }} \leq Q_{C S P, \max }^{\text {out }} \\
& 0 \leq Q_{C S P, t}^{\text {in }} \leq Q_{C S P, \text { max }}^{\text {in }}
\end{aligned}
$$

Where, $Q_{C S P \text {, max }}^{\text {out }}$ and $Q_{C S P, \max }^{\text {in }}$ represent the upper limit of heat output and input in a single period of time for the heat storage tank of CSP unit respectively.

The state of the heat storage tank:

$$
\begin{gathered}
Q_{C S P, t+1}^{H S}=Q_{C S P, t}^{H S}+Q_{C S P, t}^{\text {in }} \eta_{\text {in }}-\frac{Q_{C S P, t}^{\text {out }}}{\eta_{\text {out }}} \\
Q_{C S P, \min }^{H S} \leq Q_{C S P, t}^{H S} \leq Q_{C S P, \max }^{H S} \\
Q_{C S P, t}^{\text {in }} \cdot Q_{C S P, t}^{\text {out }}=0 \\
Q_{C S P, 1}^{H S}=Q_{C S P, 24}^{H S}
\end{gathered}
$$

Where, $Q_{C S P, t}^{H S}$ is the heat stored in the heat storage tank of CSP during period $t ; Q_{C S P, \min }^{H S}$ and $Q_{C S P, \max }^{H S}$ represent the minimum and maximum allowable heat storage capacity of the heat storage tank respectively.

\subsubsection{Turbine start-stop constraints for solar thermal units}

The heat into the steam turbine at any time period shall meet the upper limit constraint, and the total heat accumulated in that time period shall meet the heat requirements for start-up. Therefore, the constraints are established as follows:

$$
\begin{gathered}
Q_{C S P, t}^{S U} \leq y_{t}^{C S P} \cdot Q_{\max }^{\mathrm{GenIn}} \\
Q_{C S P, \text { req }}^{S U} \geq Q_{C S P, t}^{S U} \geq y_{t}^{C S P} Q_{C S P, r e q}^{S U} \\
Q_{C S P, t}^{S U}+Q_{C S P, t}^{g e n} \leq Q_{\max }^{\mathrm{GenIn}}\left(y_{t}^{C S P}+u_{t}^{C S P}\right)
\end{gathered}
$$

Where, $y_{t}^{C S P}$ is the $0-1$ variable for steam turbine start-up; $u_{t}^{C S P}$ is the $0-1$ variable for steam turbine state; $Q_{C S P, \text { req }}^{S U}$ is the heat required for steam turbine start-up.

The constraints of integer variable are listed as follows:

$$
\begin{gathered}
u_{1}^{C S P}+z_{1}^{C S P}=u_{1}^{C S P} \\
u_{t}^{C S P}-u_{t-1}^{C S P}=y_{t-1}^{C S P}-z_{t}^{C S P}, t=2, \ldots, 24 \\
0 \leq y_{t-1}^{C S P}+z_{t}^{C S P} \leq 1
\end{gathered}
$$

Where, $z_{t}^{C S P}$ is the $0-1$ variable of steam turbine shutdown. 
The generating power of CSP unit:

$$
P_{\mathrm{CSP}, t}=\kappa Q_{\mathrm{CSP}, t}^{g e n}
$$

Where, $\kappa$ and $P_{\mathrm{CSP}, t}$ are generation factor and output power of CSP.

\subsection{Heating Network}

In the heating network, the hot water is sent from the heat source to the heat exchanger on the heat load side by the water supply pipelines. After the hot water transfers heat through the heat exchanger, then it is sent back to the heat source through the return pipelines for heating again.

\subsubsection{Heat exchanger}

The model of heat exchanger at heat load is as follows:

$$
Q_{i, t}=C_{p} m_{i, t}\left(\tau_{i, t}^{N S}-\tau_{i, t}^{N R}\right)
$$

Where, $Q_{i, t}$ is the heat load of heat node $i$ at period $t ; \tau_{i, t}^{N S}$ and $\tau_{i, t}^{N R}$ respectively represent the node supply temperature and the node return temperature; $C_{p}$ is the specific heat capacity of heat medium in pipeline; $m_{i, t}$ is the mass ow rate of heat node $i$ at period $t$.

\subsubsection{Heat Source}

The model of heat source is as follows:

$$
Q_{t}^{S}=C_{p} m_{S, t}\left(\tau_{S, t}^{N S}-\tau_{S, t}^{N R}\right)
$$

Where, $Q_{t}^{S}$ is the supply heat power of the heat source at period $t ; \tau_{S, t}^{N S}$ and $\tau_{S, t}^{N R}$ respectively represent the heat source node supply temperature and the node return temperature; $m_{S, t}$ is the mass ow rate of heat source node at period $t$.

\subsubsection{Temperature Mixing}

Mass owing into the same node is mixed, and the temperature of the mixed mass is as follows:

$$
\begin{gathered}
\sum\left(\tau_{j}^{\text {S,out }} \cdot m_{j-i}^{\text {in }}\right)=\tau_{i}^{N S} \cdot \sum m_{j-i}^{\text {in }} \\
\sum\left(\tau_{j}^{R, \text { in }} \cdot m_{i-j}^{\text {out }}\right)=\tau_{i}^{N R} \cdot \sum m_{i-j}^{\text {out }} \\
\sum m_{i-j}^{\text {out }}=\sum m_{j-i}^{\text {in }}
\end{gathered}
$$

Where, $\tau_{j}^{S, \text { out }}$ and $\tau_{j}^{R, \text { in }}$ are mass flow temperature at the outlet of pipeline $j$ in the supply network and mass flow temperature at the inlet of pipeline $j$ in the return network; $m_{j-i}^{i n}$ is the mass flow rate from pipeline $j$ to heat node $i ; m_{i-j}^{\text {out }}$ is the mass flow rate from heat node $i$ to pipeline $j$.

\subsubsection{Temperature Drop}

The node temperature of water supply pipeline:

$$
\tau_{j}^{S, \text { out }}=\left(\tau_{j}^{S, i n}-\tau_{a m}\right) \mathrm{e}^{-\frac{\lambda d_{j}}{C_{p} m_{j}}}+\tau_{a m}
$$

Where, $\tau_{j}^{\text {S, in }}$ is mass flow temperature at the inlet of pipeline $j$ in the supply network; $\tau_{a m}$ is ambient temperature; $\lambda$ is heat transfer coefficient per unit length of pipeline; $d_{j}$ is the length of pipeline $j ; m_{j}$ is the mass flow rate of pipeline $j$. 
The relationship between the inlet and outlet temperatures of the water supply network is shown in (23). After taking the heat loss of the return water network into account, the relationship between the inlet and outlet temperatures of the return water network is as follows:

$$
\tau_{j}^{R, o u t}=\left(\tau_{j}^{R, i n}-\tau_{a m}\right) \mathrm{e}^{\frac{\lambda d_{j}}{C_{p m j}}}+\tau_{a m}
$$

Where, $\tau_{j}^{R, o u t}$ and is mass flow temperature considering temperature drop at the outlet of pipeline $j$ in the return network.

\subsubsection{Circulating Water Pump}

Circulating water pump maintains heat medium flow in pipes, its model is as follows:

$$
P_{t}^{C W P}=\frac{Q_{t}^{C W P}}{Q_{\max }^{C W P}} \cdot P_{\max }^{C W P}
$$

Where, $P_{t}^{C W P}$ is the electric power consumed by circulating water pump at period $t ; P_{t}^{C W P}$ is the heat energy transmitted by the circulating water pump at period $t ; Q_{\max }^{C W P}$ and $P_{\max }^{C W P}$ are respectively the maximum heat energy that can be transmitted by the circulating water pump and rated power of circulating water pump.

\subsection{Model of Gas Turbine}

As the main equipment to provide electric energy in IES, the efficiency of gas turbine is greatly affected by unit output, and will decrease with the decrease of unit output. In this paper, efficiency of gas turbine and the corresponding gas consumption of gas turbine are shown as follows:

$$
\begin{gathered}
\eta_{G T, t}^{i}=a_{i}+b_{i} P_{G T, t}^{i *}+c_{i}\left(P_{G T, t}^{i *}\right)^{2}+d_{i}\left(P_{G T, t}^{i *}\right)^{3} \\
F_{G T, t}^{i}=\frac{P_{G T, t}^{i *}}{\eta_{G T, t}^{i}}
\end{gathered}
$$

Where, $\eta_{G T, t}^{i}$ is the efficiency of gas turbine $i$ at period $t ; P_{G T, t}^{i}$ and $P_{G T, t}^{i *}$ are the unit values of generating power and generating power of gas turbine $i$ at period $t ; F_{G T, t}^{i}$ is the gas consumption of gas turbine $i$ at period $t ; a_{i}, b_{i}, c_{i}, d_{i}$ is the efficiency coefficient of gas turbine $i$.

The carbon emission of GT is directly proportional to the power generated:

$$
T_{G T, t}^{i}=\lambda_{G T, t}^{i} P_{G T, t}^{i}
$$

Where, $\lambda_{G T, t}^{i}$ is the carbon emission coefficient of the gas turbine $i$; $T_{G T, t}^{i}$ is the carbon emission of gas turbine $i$ during period $t$.

\subsection{Model of Gas Boiler}

The relationship between heat production power and gas consumption of gas fired boiler is as follows:

$$
Q_{G B, t}=F_{G B, t} \cdot \eta_{G B}
$$

Where, $Q_{G B, t}$ and $F_{G B, t}$ are the heat production power and gas consumption of the gas boiler during time period $t ; \eta_{G B}$ is the efficiency of the gas boiler. 
6 of 10

\subsection{Model of Waste Heat Boiler}

The waste heat boiler can centrally recover the heat generated by the gas turbine, and the heat power it can provide is as follows:

$$
Q_{W H, t}=\left[\sum_{i=1}^{n_{G T}} F_{G T, t}^{i}\left(1-\eta_{G T, t}^{i}\right)\right] \eta_{W H}
$$

Where, $Q_{W H, t}$ is the output heat power of waste heat boiler during time period $t ; \eta_{W H}$ is the efficiency of waste heat boiler.

\subsection{Heat Storage Tank}

The heat storage tank can be used to improve the flexibility of CHP units. It is often used to decouple the rigid constraints of "determining electricity by heat". The decoupling time is related to the capacity of the heat storage tank, and the decoupling ability is related to the charging / discharging power of the heat storage tank [12]. Heat storage tank usually has good thermal insulation, so the heat dissipation loss of heat storage tank is ignored in this model. The model of heat storage tank:

$$
\begin{gathered}
Q_{t+1}^{\mathrm{HS}}=Q_{t}^{\mathrm{HS}}+Q_{\mathrm{in}, t}^{\mathrm{HS}}-Q_{\text {out }, t}^{\mathrm{HS}}, \quad t=1, \ldots, 23 \\
0 \leq Q_{t}^{\mathrm{HS}} \leq Q_{\max }^{\mathrm{HS}} \\
0 \leq Q_{\mathrm{in}, t}^{\mathrm{HS}} \leq Q_{\mathrm{in}, \max }^{\mathrm{HS}} \\
0 \leq Q_{\text {out }, t}^{\mathrm{HS}} \leq Q_{\mathrm{out}, \max }^{\mathrm{HS}} \\
Q_{\mathrm{in}, t}^{\mathrm{HS}} \cdot Q_{\mathrm{out}, t}^{\mathrm{HS}}=0
\end{gathered}
$$

Where, $Q_{\mathrm{in}, t}^{\mathrm{HS}}$ and $Q_{\mathrm{out}, t}^{\mathrm{HS}}$ respectively represent the thermal storage / release power of the heat storage tank at time $t ; Q_{\mathrm{in}, \max }^{\mathrm{HS}}$ and $Q_{\mathrm{out}, \max }^{\mathrm{HS}}$ represent the maximum heat storage power and heat release power of the heat storage tank at time $t ; Q_{t}^{\mathrm{HS}}$ and $Q_{\max }^{\mathrm{HS}}$ represent the heat storage capacity of the heat storage tank at time $t$ and the maximum heat storage capacity of the heat storage tank.

\section{Low-Carbon Economical Dispatch of IES}

\subsection{Objective Function}

The low-carbon economic dispatch model established in this paper targets at minimizing total operating costs, which can be defined as follows:

$$
F=\min \left(C_{\text {grid }}+C_{\text {gas }}+C_{\text {eq }}+C_{\mathrm{ab}}+C_{\mathrm{w}}\right)
$$

Where the five terms donate the interaction costs with external power grids, gas purchase costs, operating costs of various equipment in the IES system, cost of abandoning wind and solar energy, and penalty costs for carbon emissions, respectively.

The costs of interaction between the system and the external grid can be expressed as the costs of electricity purchase minus the revenue:

$$
C_{\text {grid }}=\sum_{t=1}^{24}\left(\gamma_{\text {grid }, t}^{\text {buy }} P_{\text {grid }, t}^{\text {buy }}-\gamma_{\text {grid }, t}^{\text {sell }} P_{\text {grid }, t}^{\text {sell }}\right)
$$

Where $\gamma_{\text {grid, }, t}^{\text {buy }}$ and $\gamma_{\text {grid, }, t}^{\text {sell }}$ represent the electricity purchase and sale price during $t$ period, respectively; $P_{\text {grid }, t}^{\text {buy }}$ and $P_{\text {grid }, t}^{\text {sell }}$ represent the quantity of electricity purchased and electricity sold during $t$ period, respectively. 
The gas purchase costs can be defined as follows:

$$
C_{\text {gas }}=\sum_{t=1}^{24} \gamma_{\text {gas }, t} G_{t}^{\text {total }}
$$

Where $\gamma_{\text {gas, } t}$ refers to the gas purchase price during $t$ period; $G_{t}^{\text {total }}$ refers to the quantity of gas purchased during $t$ period.

The operating costs of various equipment in the IES system can be described as follows:

$$
C_{\mathrm{eq}}=\sum_{t=1}^{24}\left(\gamma_{\mathrm{PV}} P_{\mathrm{PV}, t}+\gamma_{\mathrm{WP}} P_{\mathrm{WP}, t}+\gamma_{\mathrm{CSP}} P_{\mathrm{CSP}, t}\right)
$$

Where $\gamma_{\mathrm{PV}}, \gamma_{\mathrm{WP}}$ and $\gamma_{\mathrm{CSP}}$ are the operating cost coefficients of photovoltaic power, wind power and CSP unit, respectively; $P_{\mathrm{PV}, t}$ and $P_{\mathrm{WP}, t}$ are the power of photovoltaic power and wind power.

The cost of abandoning wind and solar energy can be described as follows:

$$
C_{\mathrm{ab}}=\sum_{t=1}^{24}\left(\gamma_{\mathrm{ab}}\left[\left(\bar{P}_{\mathrm{WP}, t}-P_{\mathrm{WP}, t}\right)+\left(\bar{P}_{\mathrm{PV}, t}-P_{\mathrm{PV}, t}\right)\right]\right)
$$

Where, $\gamma_{\mathrm{ab}}$ is the penalty coefficient of abandoning wind and solar; $\bar{P}_{\mathrm{WP}, \text { and }} \bar{P}_{\mathrm{PV}, t}$ are respectively the predicted output power of wind power and photovoltaic power during $t$ period.

The penalty costs for carbon emissions can be described as follows:

$$
C_{\mathrm{w}}=\gamma_{\mathrm{w}} \sum_{t=1}^{24}\left(\lambda_{\mathrm{GT}} P_{\mathrm{GT}, t}+\lambda_{\text {grid }} P_{\text {grid }, t}^{\mathrm{buy}}+\lambda_{\mathrm{GB}} Q_{\mathrm{GB}, t}\right)
$$

Where, $\gamma_{\mathrm{w}}$ is the carbon emission penalty coefficient; $\lambda_{\text {grid }}$ and $\lambda_{\mathrm{GB}}$ are respectively the carbon emission coefficient of thermal power generating units which provide the purchased electricity of IES and the carbon emission coefficient of gas boilers.

\subsection{Constraints}

The proposed model is subject to operation constraints of four parts, namely electric power constraints, heating power constraints, natural gas load constraints and unit constraints.

The electric power constraints are listed as follows:

$$
\begin{aligned}
P_{\mathrm{PV}, t}+P_{\mathrm{WP}, t}+P_{\mathrm{GT}, t}+P_{\text {grid }, t}^{\text {buy }}+P_{t}^{\mathrm{CSP}}= & P_{t}^{\text {load }}+P_{\text {grid }, t}^{\text {sell }} \\
& +P_{t}^{C W P}
\end{aligned}
$$

Where, $P_{t}^{\text {load }}$ is the electric load demand of the IES.

The heating power constraints are listed as follows:

$$
Q_{\mathrm{GB}, t}^{\text {therm }}+Q_{\mathrm{WH}, t}^{\text {therm }}=Q_{\text {load }, \mathrm{t}}^{\text {therm }}+Q_{t}^{\mathrm{D}}
$$

Where, $Q_{\mathrm{GB}, t}^{\text {therm }}$ and $Q_{\mathrm{WH}, t}^{\text {therm }}$ are respectively the heating power provided by the gas boiler and the waste heat boiler during the $t$ period; $Q_{\text {load }, t}^{\text {therm }}$ is the heating load requirements of the system during the $t$ period; $Q_{t}^{\mathrm{D}}$ represents the waste heat of heating network during the $t$ period.

The natural gas load constraints are listed as follows:

$$
G_{t}^{\text {total }}=G_{\mathrm{GT}, t}+G_{\mathrm{GB}, t}
$$


Where $G_{\mathrm{GT}, t}$ and $G_{\mathrm{GB}, t}$ represent the gas consumption of gas turbine and gas boiler during $t$ period, respectively.

As for unit constraints, their details can be found in [15].

\section{Case Studies}

In this paper, a 9-bus power system [13] and 8-bus thermal system [14] shown in Fig. 1 is analysed. In this example, there are 3 gas turbines, one set of wind and photovoltaic power generation units, two gas boilers and one waste heat boiler. For the economic and technical parameters of the equipment mentioned above, which refer to the data in [15]. The case studies are done on a laptop with four processors at $2.80 \mathrm{GHz}$ with $16 \mathrm{~GB}$ of memory. The programs are developed using Matlab R2020a, and all MILPs and LPs are solved with the solvers of Gurobi 9.1 [16]. The relative gap of the MILP solver is $0.1 \%$.

\subsection{Power Supply and Loss In IES}

The electric power in IES is mainly supplied by gas turbines and power generation equipment, and is consumed by electric loads and power-consuming components. The real-time electric power of the system is shown in Fig. 2.

It can be seen from Fig. 2 that the IES system in the calculation example can meet the electrical load demand of the system on that day. Moreover, this form of solar energy utilization has an advantage that other forms of solar energy conversion can't match. That is, the water heated by solar energy can be stored in a heat storage, which can still drive the steam turbine a few hours after the sun sets. Between 0:00 and 8:00, because the amount of light is very small or zero, the PV power generation during this period is very small. The heat generated by CSP is stored in HS, and when the amount of light is small or zero, it can still drive the steam turbine to generate electricity. At the same time, because the cost of purchasing electricity from the grid is relatively small, at this time, the electric load in the system obtains a larger proportion of the electricity purchased from the external grid After 8:00, with the increase in PV output and the increase in power purchase price, GT units began to increase output and maintain supply power of a high level to meet the electrical load demand in the system and the system purchases power from the external grid changes with the electrical load demand fluctuations to ensure that the economic cost required to maintain electrical power balance is minimized.

\subsection{Thermal Power Supply and Loss in IES}

The thermal power in the IES system is mainly provided by GB, WH units and HS, and consumed by the thermal load. The real-time thermal power of the system is shown in Fig. 3.

It can be seen from Fig. 3 that the IES system meets the heat load demand for that day within the planned time, but it can be clearly seen in the figure that the heat power of the system exceeds the heat load demand during the period of 8:00-24:00. There is a certain heat loss power situation. The WH unit always provides heat power to the system, that is the gas turbine unit heat and power cogeneration, and the part that is not enough to meet the heat load is provided by GT. HS charges when the heating power is excessive, and discharges when the heating power is insufficient.

\subsection{Real-time Situation of HS in CSP and Heat Network}

The charging and discharging heat of HS in CSP in each scheduling period is shown in Fig. 4. Based on Fig. 4: during 0:00-4:00 and 18:00-24:00, the HS in CSP is exothermic, the thermal power HS giving out is used for generating electricity. And during 7:00-8:00, 11:00-12:00 and 15:00-17:00, the HS in CSP is endothermic, thermal power is stored into the HS. The thermal power of HS remains a constant during 5:00-6:00, 9:00-10:00 and 12:00-14:00 period.

Fig. 5 demonstrates the charging and discharging situation of HS in heat grid in each scheduling period. Based on Fig. 5: during 4:00-8:00, 12:00-15:00, 17:00-18:00 and 21:00-22:00 the HS is charging. 
During 0:00-3:00, 9:00-11:00, 16:00-17:00, 19:00-20:00 and 23:00-24:00 the HS is discharging. It can be obtained that the HS helps regulating peak of the system, which means HS would charge when the thermal power is surplus and it would discharge when the thermal power is insufficient. This illustrates that the HS in heat grid can regulate the peak and reduce the total operating cost. Therefore, it's of necessity to add HS in the proposed model.

\subsection{HS in CSP and Capacity of CSP Unit}

According to Fig. 6, when the capacity of HS in CSP unit increases, the cost decreases slightly from 66782.3 yuan to 66032.4 yuan. With respect to the capacity of CSP unit, the cost drops sharply from 67892.1 yuan to 62981.6 yuan with the increment of the capacity of CSP unit. Fig.6 proves that larger the capacity of HS and CSP unit is, the lower the cost would be. Therefore, increasing the capacity of HS appropriately helps reduce the total cost.

\subsection{Relationship between the HS in DHS and the Cost of IES}

According to Fig. 7, the total operating cost of the proposed model decreases with the increment of the capacity of HS, which illustrates HS has the potential to reduce the total operating cost. Therefore, it can be obtained that HS is essential in the model.

\subsection{Analysis with CSP and without CSP}

It can be seen from Table I that the total cost of IES with CSP is reduced from 6,7892.1 yuan to 6,6032.4 yuan compared with the IES without CSP. Because the CSP plant participates in the IES, although the operating cost of equipment is increased, but more the electricity purchasing cost, gas purchasing cost and penalty costs for carbon emissions are effectively reduced. The utilization rate of new energy in the system is effectively improved, thus saving non-renewable energy such as gas boiler power and promoting the economy of the system.

Table 1. Analysis with CSP and without CSP

\begin{tabular}{ccc}
\hline Cost & With CSP & Without CSP \\
\hline$C_{\text {grid }}$ & 13563.31yuan & 15193.02yuan \\
$C_{\text {gas }}$ & 39972.48yuan & 40206.11yuan \\
$C_{\text {eq }}$ & $1252.429 y u a n$ & $1034.5 y u a n$ \\
$C_{\mathrm{ab}}$ & 0 & 0 \\
$C_{\mathrm{w}}$ & 11244.2 yuan & 11458.49 yuan \\
Total Cost & 66032.42 yuan & $67892.13 y u a n$ \\
\hline
\end{tabular}

\section{Conclusion}

An Electricity-Heat IES model is presented in this paper, which focuses on the concentrating solar power unit and heat loss in the DHN. The simulation results for the nine-bus and eight-node system indicate that the proposed low-carbon economical dispatch model can meet the electrical and thermal load requirements of the system, increase energy diversity in IES and reduce the operating cost of the system. An example is given to verify the operation cost of the system with or without CSP unit, and the effectiveness of CSP unit participating in the system operation is verified.

Based on the proposed low-carbon economical dispatch model of Electricity-Heat IES, several interesting directions are open for future study. How to design system operation scheme under uncertainty of solar and wind power is still a note-worthy topic. Because of CSP unit's small capacity, its large-scale integration would involve an extensive number of units which can bring about numerous variables and constraints to the problem, a clustered CSP model would be helpful to reduce the calculation complexity of the model, which is an interesting issue that needs more study. Another 
important but challenging topic is the bidding planning of energy market considering CSP unit. All these issues mentioned above are worth further investigating.

\section{References}

1. Y. Wang, B. Zeng, J. Guo, et al, "Multi-energy flow calculation method for integrated energy system containing electricity heat and gas," Power System Technology, 2016, vol. 10, pp. 2942-2951.

2. Y. Li, W. Liu, J. Zhao, et al, "Optimal dispatch of combined electricity-gas-heat energy systems with power-to-gas devices and benefit analysis of wind power accommodation," Power System Technology, 2016, vol. 12, pp. 3680-3689.

3. Y. Xiong, J. Wu , Q. Wang and X.Mao, “An Optimization Coordination Model and Solution for Combined Cooling, Heating and Electric Power Systems With Complimentary Generation of Wind, PV, Gas and Energy Storage," Proceedings of the CSEE, 2015, vol. 14, pp. 3616-3625.

4. W. Gu, S. Lu, J.Wang, et al, "Modeling of the Heating Network for Multi-district Integrated Energy System and Its Operation Optimization," Proceedings of the CSEE, 2017, vol. 5, pp. 1305-1315.

5. B. Bohm, S. Ha, W. Kim, et al, Simple models for operational optimization, Kongens Lyngby, Denmark:Technical University of Denmark (DTU) , 2002.

6. X.Liu, N. Jenkins, J. Wu, et al, Combined analysis of electricity and heat networks, Cardiff, UK: Cardiff University, 2014.

7. X. Wang, L.Zhao, P. Zou, et al, "Topology structure of spatial heat-supply network based on graph theory," Chinese Journal of Computational Physics, 2014, vol. 2, pp. 207-215.

8. Z. Gu, C. Kang, X. Chen, et al, "Operation optimization of integrated power and heat energy systems and the benefit on wind power accommodation considering heating network constraints," Proceedings of the CSEE, 2015, vol. 14, pp. 3596-3604.

9. R. Chen, H. Sun, Z. Li,et al, "Grid dispatch model and interconnection benefit analysis of concentrating solar power plants with thermal storage," Automation of Electric Power Systems, 2014, vol. 19, pp. 1-7.

10. R. Domínguez, A.J. Conejo, M. Carrión, “Operation of a fully renewable electric energy system with CSP plants," Applied Energy,2014, vol. 119, pp. 417-430.

11. J. Wang, J. Shi, F. Wen, et al, “Optimal operation of CHP micro grid with concentrating solar power plants considering demand response," Automation of Electric Power Systems, 2019, vol. 1, pp. 176-189.

12. C. Wu, Modeling and optimal operation of power and heat integrated energy system, Nanjing, China: Southeast University, 2019.

13. G. Huang, W. Liu, F. Wen, et al, “Collaborative planning of integrated electricity and natural gas energy systems with power-to-gas stations," Electric Power Construction, 2016, vol. 9, pp. 1-13.

14. R. Li, L. Chen, T. Yuan and C. Li, "Optimal dispatch of zero-carbon-emission micro Energy Internet integrated with non-supplementary fired compressed air energy storage system," J. Mod. Power Syst. Clean Energy, 2016, vol. 4, pp. 566-580.

15. N. Zhang, X. Jian, Y. Ding and Y. Wang, “Day-ahead Low-Carbon Economical Dispatch of Integrated Energy System With CSP and ORC Based on Improved Imperialist Competitive Algorithm," unpublished.

16. Gurobi Optimization, Inc.. (2020). Gurobi Homepage [Online]. Available: http://www.gurobi.com/ 